\title{
REARRANGEMENTS IN STEADY VORTEX FLOWS WITH CIRCULATION
}

\author{
ALAN R. ELCRAT AND KENNETH G. MILLER
}

(Communicated by Barbara L. Keyfitz)

\begin{abstract}
It is shown that a steady two-dimensional flow, in which a finite vortex is in equilibrium with the irrotational flow past an obstacle, can be obtained as the solution of a variational problem in the class of rearrangements of a fixed function in $L^{p}$. The main step is to establish a bound on the support of the vorticity. The advantage of this approach, as in the recent works of Burton, Benjamin, and Auchmuty, is that the profile function of the vorticity is determined by the rearrangement class in which solutions are sought.
\end{abstract}

\section{INTRODUCTION}

The idea that for steady, inviscid flows the vorticity should give energy an extreme value goes back to Kelvin. More recently it has been suggested by Benjamin [3] and the work of Arnold [1] that a natural class in which to seek solutions is the set of rearrangements of a fixed function. In particular, the vorticity in a time-dependent flow is a rearrangement of its initial values. Only recently has the required functional analysis for proving existence in a class of rearrangements been worked out. We cite in particular Burton [5], [6] and an unpublished manuscript of Auchmuty and Benjamin [2]. We will show here that these ideas can be applied to a problem recently studied by the authors [7] in which existence was shown for a finite vortex in equilibrium with an irrotational flow with circulation around an obstacle. That work builds on earlier work of Turkington [8]. An essential ingredient in both is a "local support" lemma which is required in order to obtain a solution of the Euler equations from a solution to the variational problem. In this paper, after pointing out that Burton's results [5] can be applied to this problem to establish the existence of a maximizer of the appropriate functional on a class of rearrangements, we will adapt the methods of Turkington [8] to show that this maximizer provides a solution to the Euler equations by proving the needed local support lemma. The work of Turkington [9] on vortex pairs behind a symmetric obstacle can be similarly extended to the context of rearrangements.

Received by the editors September 1, 1989 and, in revised form, March 5, 1990.

1980 Mathematics Subject Classification (1985 Revision). Primary 35Q05, 76C05.

Research of the first author partially supported by U.S. Air Force Grant AFOSR-86-0274. 
Let $D$ be an exterior plane domain bounded by a Jordan curve and $\eta$ the stream function for an irrotational flow in $D$ which is uniform at $\infty$ and has circulation $\Gamma$ around $\partial D$. Let $g$ be the Green's function for Laplace's equation on $D$ satisfying, as in [7],

$$
\int_{\partial D} \frac{\partial g}{\partial n}(x, y) d s_{x}=0, \quad \text { for all } y \in D
$$

$g(x, y)=C(y)$ for $x \in \partial D$ and $h(x, y)=-g(x, y)-(1 / 2 \pi) \log |x-y|$, converging to 0 as $y \rightarrow \infty$. By the classic Kirchoff-Routh theory a point $x_{0} \in D$ is a stable equilibrium position for a point vortex in a flow, with circulation $k$ about the vortex and circulation $\Gamma$ about $\partial D$, if the Routh function

$$
H(x)=\frac{1}{2} k h(x, x)-\eta(x)
$$

has a strict local minimum at $x_{0}$. We seek solutions to the Euler equations in $D$ for which the vorticity is concentrated "near" such a local minimum $x_{0}$.

Let $S$ be a compact subregion of $D$ with $x_{0}$ in its interior such that $H$ has a strict minimum on $S$ at $x_{0}$. Let $f \in L^{p}\left(\mathbb{R}^{2}\right), p>1$, be compactly supported, $f \geq 0, \int_{D} f=k$. A function $g$ is said to be a rearrangement of $f$ if $\left|f^{-1}([\alpha, \infty))\right|=\left|g^{-1}([\alpha, \infty))\right|$ for all $\alpha \in \mathbb{R}$, where $|A|$ denotes the Lebesgue measure of the set $A$. For $\lambda>0$ define $f_{\lambda}(x)=\lambda f\left(\lambda^{1 / 2} x\right)$. Let $K_{\lambda}$ be the set of rearrangements $\omega$ of $f_{\lambda}$ such that supp $\omega \subseteq S$. We assume that $\lambda$ is large enough that $K_{\lambda} \neq \varnothing$. Note that for any $\omega \in K_{\lambda}$, the circulation

$$
\int_{D} \omega=k
$$

and

$$
\|\omega\|_{p}=\lambda^{1 / q}\|f\|_{p},
$$

where $q$ is the conjugate exponent, $p^{-1}+q^{-1}=1$.

Consider the functional

$$
E(\omega)=\frac{1}{2} \int_{D} G \omega(x) \omega(x) d x+\int_{D} \eta(x) \omega(x) d x,
$$

where $G$ is the integral operator with kernel $g$. $G$ is a compact symmetric operator from $L^{p}(S)$ to $L^{q}(S)$. Burton [6, Corollary 3.4] implies that $E$ assumes a maximum on $K_{\lambda}$, and, moreover, if $\omega$ is a maximizer, then $\omega=\phi(G \omega+\eta)$ for $x \in S$ for some increasing function $\phi$.

Throughout the following, $\omega=\omega_{\lambda}$ is the maximizer of $E$ on $K_{\lambda}$ and $\Omega=$ $\operatorname{supp} \omega$. By the last statement of the preceding paragraph

$$
\Omega=\{x \in S: G \omega(x)+\eta(x) \geq \mu\}
$$

for some real number $\mu=\mu_{\lambda}$. Define

$$
\psi=G \omega+\eta-\mu, \quad \text { and } \quad T(\omega)=\frac{1}{2} \int_{D} \psi \omega=\frac{1}{2} \int_{D} \psi^{+} \omega .
$$

$C$ will denote any constant independent of $\lambda$. $C$ may depend on $f$ and $p$. 
Lemma 1. $T(\omega) \leq C$.

Proof. Let $S_{1}$ be a compact subset of $D$ such that $S \subset \operatorname{int} S_{1}$. Then there is a $\delta$, independent of $\lambda$, such that $\psi(x)<\delta$ for all $x \in \partial S_{1}$. Let $\psi_{1}=\psi-\delta$ and $T_{1}(\omega)=\frac{1}{2} \int \psi_{1}^{+} \omega$. Since

$$
T(\omega) \leq T_{1}(\omega)+\delta k / 2,
$$

it suffices to show that $T_{1}(\omega) \leq C$. Since $\psi_{1}<0$ near $\partial S_{1}$, Hölder's inequality and the Sobolev embedding theorem imply that

$$
\left\|\psi_{1}^{+}\right\|_{q} \leq C\left|\Omega_{1}\right|^{1 / q}\left\|\nabla \psi_{1}^{+}\right\|_{2},
$$

where $\Omega_{1}=\operatorname{supp} \psi_{1}^{+}$. Also,

$$
\left|\Omega_{1}\right| \leq|\Omega|=\left|\operatorname{supp} f_{\lambda}\right|=C \lambda^{-1} .
$$

Therefore, using (2),

$$
2 T_{1}(\omega) \leq\left\|\psi_{1}^{+}\right\|_{q}\|\omega\|_{p} \leq C\left\|\nabla \psi_{1}^{+}\right\|_{2} .
$$

An integration by parts shows that

$$
\left\|\nabla \psi_{1}^{+}\right\|_{2}=\left(2 T_{1}(\omega)\right)^{1 / 2},
$$

and the lemma follows.

Lemma 2 (Support lemma). There exist $C$ and $\lambda_{0}$ such that the diameter of $\Omega \leq C \lambda^{-1 / 2}$ for all $\lambda>\lambda_{0}$.

Proof. Let $\omega^{*}$ be the symmetric rearrangement of $f_{\lambda}$ around the point $x_{0}$. Let $\varepsilon$ be the radius of the support of $f_{\lambda}$. Note that $\varepsilon=C \lambda^{-1 / 2}$. If $\lambda$ is sufficiently large, $\omega^{*} \in K_{\lambda}$. Hence, by the same argument as in Lemma 3.1 of Turkington [8],

$$
E(\omega) \geq E\left(\omega^{*}\right) \geq-\left(k^{2} / 4 \pi\right) \log (2 \varepsilon)-k H\left(x_{0}\right)+o(1)
$$

as $\lambda \rightarrow \infty$. The identity

$$
E(\omega)=T(\omega)+\left(\frac{k}{2}\right) \mu+\frac{1}{2} \int \eta \omega
$$

and Lemma 1 imply that

$$
\mu \geq-\left(\frac{k}{2 \pi}\right) \log \varepsilon-C .
$$

Let $x \in \Omega$. Now $G \omega+\eta \geq \mu$ on $\Omega$ and the boundedness of $h(x, y)$ and $\eta(x)$ on $S$ imply

$$
\frac{1}{2 \pi} \int_{D} \log |x-y|^{-1} \omega(y) d y \geq C+\mu .
$$

Combining with (4) gives

$$
\frac{1}{2 \pi} \int_{D} \log \frac{\varepsilon}{|x-y|} \omega(y) d y \geq-C .
$$


Let $B_{r}$ denote the disk of radius $r$ about $x$. For any $R \geq 1$ and $x \in \Omega$,

$$
\int_{B_{R e}} \log (\varepsilon /|x-y|) \omega(y) d y \leq\|\omega\|_{p}\left\|\log (\varepsilon /|y|)^{+}\right\|_{q} \leq C,
$$

since $\|\omega\|_{p} \leq C \lambda^{1 / q}$ and $\left\|\log (\varepsilon /|y|)^{+}\right\|_{q}^{q} \leq C_{q} \varepsilon^{2} \leq C \lambda^{-1}$. Inequalities (5) and (6) imply that

$$
\int_{D-B_{R \varepsilon}} \omega(x) d x \leq C(\log R)^{-1}<k / 2
$$

for $R$ sufficiently large. Since $\int \omega=k$, it follows that diameter $\Omega \leq 2 R \varepsilon$ for some $R$. This proves the lemma.

Theorem. If $\lambda$ is sufficiently large, then the maximizer $\omega$ of $E$ on $K_{\lambda}$ is the vorticity and $\psi=G \omega+\eta-\mu$, the stream function for a solution of the Euler equations in $D$ with uniform velocity at $\infty$ and satisfying $\psi=$ constant on $\partial D, \int_{\partial D}(\partial \psi / \partial n) d s=\Gamma$.

Proof. That the stated boundary conditions are satisfied follows immediately from the properties of $g$ and $\eta$. According to the variational principle of Arnold [1] as used in [7], [8], and [9], $\omega$ and $\psi$ are the vorticity and stream function for a solution to the Euler equations if $\omega$ is a maximizer of $E$ over some class $K \subset L^{p}(D)$ such that $K$ contains $\omega \circ \xi$ for area-preserving diffeomorphisms $\xi$ of $D$ sufficiently near the identity (see [7, Proposition 4.1]). As in [7] and [8], this will follow from Lemma 2 if we prove that $x_{\lambda}=\frac{1}{k} \int_{D} x \omega_{\lambda}(x) d x$ converges to $x_{0}$ as $\lambda \rightarrow \infty$.

Define functionals $I$ and $F$ on $K_{\lambda}$ by

$$
\begin{aligned}
I(\zeta) & =\frac{1}{2} \int_{D} \int_{D} h(x, y) \zeta(x) \zeta(y) d x d y-\int_{D} \eta(x) \zeta(x) d x \\
F(\zeta) & =\frac{1}{4 \pi} \int_{D} \int_{D} \log |x-y|^{-1} \zeta(x) \zeta(y) d x d y .
\end{aligned}
$$

Then $I(\zeta)=F(\zeta)-E(\zeta)$. Let $\omega_{\lambda}^{*}$ be the symmetric rearrangement of $\omega_{\lambda}$ about the point $x_{0}$. An $n$-dimensional generalization [4] of an inequality of $F$. Riesz on rearrangements implies $F\left(\omega_{\lambda}^{*}\right) \geq F\left(\omega_{\lambda}\right)$. Therefore $I\left(\omega_{\lambda}\right) \leq I\left(\omega_{\lambda}^{*}\right)$. Letting $\bar{x}$ be a limit point of $x_{\lambda}$, it follows that $H(\bar{x}) \leq H\left(x_{0}\right)$. Hence $x_{\lambda}$ converges to $x_{0}$ as $\lambda \rightarrow \infty$.

Remark. The flow obtained may be thought of in relation to solutions of the time-dependent problem in which the initial data for the vorticity is a function generating the rearrangement class $K_{\lambda}$. Since the minimizer of the Routh function is a stable equilibrium of the time evolution of a point vortex, it is reasonable to assume that the same is true of the equilibrium solution obtained here. A complete proof is not available, but one might be given on the basis of the ideas in [10] where flow in a bounded domain without an external flow is considered. Then initial data in $K_{\lambda}$ near to the equilibrium solution $\omega$ would yield a solution which remains close to $\omega$ for all time and has the same vorticity 
distribution as $\omega$. One might even conjecture that if the centroid of the initial data equals that of $\omega, \omega$ is the limit as time approaches infinity of the solution of the initial value problem. In any case, there are as many equilibrium solutions as there are distinct classes $K_{\lambda}$, and their relationship to time-dependent flows may be thought of in light of the above remarks.

\section{REFERENCES}

1. V. I. Arnold, Mathematical methods of classical mechanics, Springer-Verlag, Berlin, 1978.

2. J. F. Auchmuty and T. B. Benjamin, unpublished manuscript.

3. T. B. Benjamin, The alliance of practical and analytical insights into the nonlinear problems of fluid mechanics, Applications of methods of functional analysis to problems of mechanics, Lecture Notes in Mathematics, vol. 503, Springer-Verlag, Berlin, 1976, pp. 8-29.

4. H. J. Brascamp, E. H. Lieb, and J. M. Luttinger, A general rearrangement inequality for multiple integrals, J. Funct. Anal. 17 (1974), 227-237.

5. G. R. Burton, Steady symmetric vortex pairs and rearrangements, Proc. Royal Soc. Edinburgh 108A (1988), 269-290.

6. - Variational problems on classes of rearrangements and multiple configurations for steady vortices, Ann. Inst. Henri Poincaré. Analyse Nonlineare 6 (1989), 295-319.

7. A. R. Elcrat and K. G. Miller, Steady vortex flows with circulation past asymmetric obstacles, Comm. Partial Differential Equations 12 (1987), 1095-1115.

8. B. Turkington, On steady vortex flows in two dimensions, I, Comm. Partial Differential Equations 8 (1983), 999-1030.

9. __ On steady vortex flows in two dimensions, II, Comm. Partial Differential Equations 8 (1983), 1031-1071.

10. _ On the evolution of a concentrated vortex in an ideal fluid, Arch. Rational Mech. Anal. 97 (1987), 75-87.

Department of Mathematics, The Wichita State University, Wichita, Kansas 672081595 